\title{
Noninvasive Ventilation Device-Related Pressure Injury in a Children's Hospital
}

\author{
Denise L Lauderbaugh, Rakesh Bhattacharjee, James Proudfoot, Ericka V Ortega, Lindsay A Pratt, \\ Toni L Popien, and Daniel J Lesser
}

\begin{abstract}
BACKGROUND: Noninvasive ventilation (NIV) contributes to the development of pressure injury in a significant number of hospitalized patients. Pressure injuries contribute to increased length of hospital stay, pain, infection, and disfigurement. This study examined the relationship between NIV use and pressure injuries in hospitalized subjects. METHODS: We retrospectively reviewed all patients on NIV at a tertiary-care children's hospital over a 2-y period. We studied the relationship between the characteristics of NIV use and measures of pressure injury severity. RESULTS: A total of 255 subjects, mean \pm SD age $11.3 \pm 5.8$ y with 343 episodes of NIV use were evaluated, 7.2\% $(25 / 343)$ of which were associated with pressure injury. In univariate analysis, the presence of pressure injury was associated with older age $(P=.01)$, maximum leak $(P=.01)$, 95th percentile leak $(P=.01)$, the $\log$ duration of time on NIV until pressure injury formation $(P=.01)$, and maximum inspiratory positive airway pressure level $(P=.01)$. Maximum leak remained statistically significant after multivariable analysis. CONCLUSIONS: After multivariate analysis, only high mask leak was significantly associated with developing a pressure injury. Identifying risk factors that correlate with NIV device-related hospital acquired pressure injuries in children can direct procedures to prevent pressure injury in hospitalized children at high risk. Key words: pressure injury; pressure ulcer; pediatrics; injury; hospitals; skin; noninvasive ventilation; respiratory device; device-related pressure injury. [Respir Care 2019;64(12):1455-1460. (C) 2019 Daedalus Enterprises]
\end{abstract}

\section{Introduction}

Noninvasive ventilation (NIV) is increasingly used to manage respiratory distress and sleep-disordered breathing in the pediatric in-patient setting, which broadens the incidence of undesirable effects. ${ }^{1}$ Pressure injuries occur when susceptible tissues become compressed between the bone and an external surface, which results in skin ischemia and necrosis. ${ }^{2}$ Although the epidermis is pliable and

Ms Lauderbaugh is affiliated with the Department of Respiratory Therapy, Rady Children's Hospital—San Diego, San Diego, California. Drs Bhattacharjee and Lesser are affiliated with the Division of Respiratory Medicine, Rady Children's Hospital-San Diego, University of California-San Diego, San Diego, California. Mr Proudfoot is affiliated with the Statistical Analysis of Data, Clinical and Translational Research Institute, University of California-San Diego, San Diego, California. Ms Ortega and Ms Pratt are affiliated with the Department of Nursing, Rady Children's Hospital—San Diego, San Diego, California. Ms Popien is affiliated with the Department of Respiratory Care, Rady Children's Hospital-San Diego, San Diego, California. often resistant to insult, excessive external pressure, such as that caused by NIV-mask interfaces, can cause severe injury. ${ }^{3}$

The prevention of pressure injuries and, specifically, respiratory device-related pressure injuries, has garnered increased attention by pediatric hospital safety and quality councils. ${ }^{4}$ Pressure injuries contribute to higher cost, pain, increased infection rates, disfigurement, and increased

\footnotetext{
Funding was provided through an Ellen Browning Scripps Foundation Research Scholarship.

Ms Lauderbaugh presented a version of this paper at the Editors' Choice Open Forum, held December 6, 2018, Las Vegas.
}

The authors have disclosed no conflicts of interest.

Correspondence: Denise L Lauderbaugh MPH RRT RRT-NPS, Rady Children's Hospital-San Diego, Respiratory Therapy, 3020 Children's Way, San Diego, Ca 92123. E-mail: dlauderbaugh@rchsd.org.

DOI: $10.4187 /$ respcare. 06784 
length of hospital stay. Although the absence of pressure injury is an indicator of quality care, ${ }^{5}$ The Joint Commission continues to identify prevention of pressure injuries as one of the most challenging issues hospitals face in

\section{See the Related Editorial on Page 1596}

protecting patients. ${ }^{6}$ In an internal review of pressure injuries at our institution, $>65 \%$ of these injuries were associated with medical devices such as intravenous lines, pulse oximeter probes, and nasogastric tubes. Traditionally, in-patient pressure injury prevention has fallen under the purview of nursing care, through use of regular skin assessment tools and repositioning protocols. As Florence Nightingale humbly noted: "If he has a bedsore, it's generally not the fault of the disease, but of nursing." recently, interprofessional committees and multi-disciplinary collaborations have spearheaded efforts to prevent pressure injury. ${ }^{8}$

There is a paucity of data with regard to pressure injury and NIV therapy in pediatric populations to guide these efforts. Evidence-based protocols are needed to improve the care of this high-risk group. The purpose of this study was to describe the relationship between subject demographics or NIV parameters with pressure injury. We hypothesized that both low and high patient-mask leak and higher inspiratory positive airway pressure correlate with pressure injury.

\section{Methods}

\section{Study Design}

The study (151189) was approved by the University of California-San Diego institutional review board. We used a retrospective study design to examine episodes of any use of NIV at our institution from July 1, 2013, to June 30, 2015. All patients admitted during the specified time were included if they were treated with pediatric or adult mask via a Respironics Vision (Phillips, Carlsbad, CA) ventilator. Episodes were identified by using billing codes $00110140 \mathrm{CPAP} / \mathrm{BPAP}$ on the first day and $0110142 \mathrm{CPAP} /$ BPAP on the subsequent day, and confirmed by charting data. Infants treated with NIV who were too small for a pediatric mask and required NIV via an alternate device were excluded due to the inability to accurately measure patient mask leak on this device, but we did not exclude subjects based on maximum age.

The primary diagnosis for respiratory failure that led to NIV was obtained from progress notes. At our institution, the majority of children for whom NIV is initiated to treat acute respiratory failure reside within the pediatric or cardiovascular ICUs; those who stabilized after treatment for

\section{QUICK LOOK}

\section{Current knowledge}

Continuous pressure by a medical device against the skin can cause pressure injury. Pressure injuries are preventable and can begin as early as 4-6 h of continuous pressure. Children who are medically complex and who are often prescribed noninvasive ventilation (NIV) are at higher risk for pressure injuries. Identifying risk factors for NIV-related pressure injuries could lead to strategies that prevent NIV-related pressure injury in hospitalized children.

\section{What this paper contributes to our knowledge}

Excess leak around the mask was most significantly associated with pressure injury. Monitoring of patient mask leak may serve to prevent pressure injury in children who use NIV. Subsequent prospective study designs should look to minimize mask leak as a potential strategy to reduce or eliminate NIV mask-related pressure injuries.

acute respiratory failure or who have stable sleep-disordered breathing are treated on the general ward. This study included NIV episodes that occurred at our main campus in both ICUs and general wards. NIV was discontinued and reinitiated within the same hospitalization for some individuals. To optimize quantification of the relationship between pressure injury and NIV, we identified being off NIV for $>24 \mathrm{~h}$ as a separate episode. Thus, several subjects had more than one episode recorded.

All the subjects on NIV in this study were treated with bi-level spontaneous timed or CPAP mode. Mask interfaces were chosen by the treating team and included nasal, nasal-oral, nasal pillows, and total face types. Several subjects were treated with more than one type of mask during an NIV episode, especially if they were noted to have skin redness or pressure injury. The location of pressure injury was determined and recorded. All the subjects were treated with at least one mask capable of measuring patient mask leak. All areas of the hospital are educated to follow the same skin care policy. In addition, hospital policy provides for utilization of a foam barrier between masks and the patient's skin for any patient on NIV by mask for $\geq 8 \mathrm{~h}$ per day. Commercial foam barriers are changed when visibly soiled or damp during routine (every $4 \mathrm{~h}$ ) mask removal to offload pressure and enable oral care.

\section{Study Variables}

Pressure injury was evaluated as the primary outcome in the study. Assessment of the presence of pressure injury 
Table 1. Demographic Characteristics by Subject

\begin{tabular}{|c|c|c|c|c|}
\hline \multirow{2}{*}{ Parameter } & \multicolumn{4}{|c|}{ NIV Mask-Related Pressure Injury } \\
\hline & No $(n=232)$ & Yes $(n=23)$ & Overall $(N=255)$ & $P$ \\
\hline \multicolumn{5}{|l|}{ Sex at birth, $n(\%)$} \\
\hline Female & $125(53.9)$ & $13(56.5)$ & $138(54.1)$ & \multirow[t]{2}{*}{.83} \\
\hline Male & $107(46.1)$ & $10(43.5)$ & $117(45.9)$ & \\
\hline \multicolumn{5}{|c|}{ Home NIV/CPAP, $n(\%)$} \\
\hline No & $163(70.0)$ & $12(52.0)$ & $175(68.6)$ & \multirow[t]{2}{*}{.10} \\
\hline Yes & $69(30.0)$ & $11(48.0)$ & $80(31.4)$ & \\
\hline \multicolumn{5}{|c|}{ Chronic disease, $n(\%)^{*}$} \\
\hline No & 27 (11.6) & $1(4.3)$ & $28(11.0)$ & \multirow[t]{2}{*}{.49} \\
\hline Yes & $205(88.4)$ & $22(95.7)$ & $227(89)$ & \\
\hline Age, mean \pm SD y & $10.9(5.8)$ & $14.1(5.5)$ & $11.2(5.8)$ & .01 \\
\hline
\end{tabular}

and its severity was evaluated by using the National Pressure Ulcer Advisory Panel 2009 guideline. ${ }^{9}$ The NIV mask was removed and the skin was assessed every $4 \mathrm{~h}$ by registered nurses or respiratory therapists in both general wards and ICUs. Once a pressure injury was identified, staging was made by a certified wound, ostomy, and continence nurse. Blanchable redness is reddened skin that becomes white or pale when light pressure is applied, which indicates good perfusion. This redness resolves with removal of the pressure at the point of redness. ${ }^{10}$ The National Pressure Ulcer Advisory Panel scale stages pressure injuries as follows: stage 1, nonblanchable erythema on intact skin; stage 2, partial-thickness loss with exposed dermis; stage 3, full-thickness loss; and stage 4, full-thickness loss and tissue loss, and deep tissue injury, persistent nonblanchable deep red, maroon, or purple discoloration. ${ }^{11}$ The risk of pressure injury for all the subjects was assessed by using the Braden $\mathrm{Q}$ score, a scale developed for evaluating the risk of pressure injuries. The Braden $\mathrm{Q}$ score totals from 1 to 23 ; scores $<16$ denote high risk. ${ }^{12}$

Demographic characteristics were analyzed by subject to account for repeated measures and included sex at birth, age at the time of NIV initiation, the presence of an underlying diagnosis that contributed to respiratory compromise, and use of NIV/CPAP at home. Other variables were analyzed by episode to optimize the quantification of the relationship between pressure injury and NIV use. These variables included log duration of the time on NIV, hospital stay in days, maximum inspiratory positive airway pressure, Braden Q score, and patient mask leak.

We defined patient mask leak as the total leak minus the intentional leak created by the flow through the continuous exhalation port. Intentional air leaks are part of the design of NIV circuits and necessary for $\mathrm{CO}_{2}$ removal. The intentional leak of the exhalation port is determined during the exhalation port test. This learned value is stored in the memory and is used to perform leak calculations, which ensures accuracy. ${ }^{13}$ Patient mask leak data were collected every $4 \mathrm{~h}$ as part of an NIV check from initiation to discontinuation. We determined the maximum, 95\% percentile, and minimum leak for each NIV episode.

\section{Statistical Analysis}

Data were analyzed by subject for demographic data and by NIV episode for other variables. Subject characteristics were compared according to the presence of injury by using the Mann-Whitney test for continuous variables and the Fisher exact test for categorical variables. To determine the significance of each predictor of interest and the presence of any injury, we fitted logistic mixed models and included a random intercept to account for repeated measures within subject. As a model selection procedure, we used the conditional Akaike information criterion criteria of Vaida and Blanchard ${ }^{14}$ as implemented in the $\mathrm{R}$ package cAIC4 ${ }^{15}$ to choose covariates for a multivariable logistic mixed model. We considered $P<.05$ to indicate statistical significance.

\section{Results}

We identified 255 subjects who had been on NIV 343 times. The mean $\pm \mathrm{SD}$ age was $11.2 \pm 5.8 \mathrm{y}$ (range, 2 months to $35 \mathrm{y}$ ), with 138 females $(54.1 \%)$ having been on NIV $(P=.83)$ (Table 1$)$. Although the majority of subjects were in the pediatric age range, there were 2 adults, ages 30 and $35 \mathrm{y}$, who were admitted during this time period related to the nature of their disease as being traditionally managed by pediatric specialists. The majority of NIV episodes were started due to pulmonary disease, 
Table 2. NIV and Subject Characteristics by Episode

\begin{tabular}{|c|c|c|c|c|c|}
\hline \multirow{2}{*}{ Characteristic } & \multicolumn{5}{|c|}{ NIV-Related Pressure Injury } \\
\hline & No $($ no. $=318)$ & Yes $($ no. $=25)$ & Total $($ no. $=343)$ & Odds Ratio $(95 \%$ CI) & $P$ \\
\hline Log duration NIV, mean $\pm \mathrm{SD} \mathrm{d}^{*}$ & $4.85 \pm 5.75$ & $7.15 \pm 7.40$ & $5.02 \pm 5.90$ & $1.064(0.991-1.142)$ & .01 \\
\hline Hospital length of stay, mean $\pm \mathrm{SD} d \dagger$ & $23.53 \pm 30.37$ & $24.8 \pm 27.92$ & $23.62 \pm 30.16$ & $1.004(0.988-1.021)$ & .65 \\
\hline Maximum IPAP, mean \pm SD $\quad \mathrm{cm}_{2} \mathrm{O}$ & $17.73 \pm 4.85$ & $19.84 \pm 4.41$ & $17.88 \pm 4.85$ & $1.126(1.002-1.265)$ & .01 \\
\hline \multicolumn{6}{|l|}{ Subject mask leak, mean $\pm \mathrm{SD} \mathrm{L} / \mathrm{min}$} \\
\hline Maximum & $52.46 \pm 25.75$ & $70.44 \pm 23.84$ & $53.81 \pm 26.01$ & $1.024(1.007-1.042)$ & .01 \\
\hline Mean & $31.46 \pm 12.48$ & $35.99 \pm 8.88$ & $31.8 \pm 12.30$ & $1.034(0.994-1.075)$ & .95 \\
\hline Minimum & $16.44 \pm 11.50$ & $11.32 \pm 8.19$ & $16.06 \pm 11.35$ & $0.946(0.895-1.001)$ & .054 \\
\hline 95th Percentile & $45.66 \pm 18.47$ & $57.91 \pm 14.47$ & $46.58 \pm 18.47$ & $1.039(1.012-1.066)$ & .01 \\
\hline Braden Q score, mean $\pm \mathrm{SD}$ & $17.96 \pm 3.77$ & $16.36 \pm 2.43$ & $17.84 \pm 3.71$ & $0.862(0.733-1.015)$ & .08 \\
\hline \multicolumn{6}{|l|}{ Masks used, no. (\%) } \\
\hline 1 & $184(57.9)$ & $5(20.0)$ & $189(55.1)$ & $5.784(10.930-17.335)$ & \\
\hline$>1$ & $134(42.1)$ & $20(80.0)$ & 154 (44.9) & & .01 \\
\hline \multicolumn{6}{|c|}{$\begin{array}{l}\text { Logistic mixed model, } P<.05 \text { signifies statistical significance. } \\
* \text { Median }(\mathrm{IQR}), 3.1(1.05-6.85) \\
\dagger \text { Median }(\mathrm{IQR}), 12(6-28.5) \\
\text { NIV = noninvasive ventilation } \\
\text { IPAP = inspiratory positive airway pressure } \\
\text { IQR = interquartile range }\end{array}$} \\
\hline
\end{tabular}

with pneumonia being the most frequent primary diagnosis. Of 343 NIV episodes studied, 25 (7.3\%) incurred pressure injury.

Pressure injury stages varied, with most injuries (64\%) graded as stage 1 . The nasal bridge (64\%), cheeks $(32 \%)$, and chin $(4 \%)$ were the most commonly injured sites. Twenty-three subjects developed pressure injuries, and 2 had more than one event with a documented pressure injury. Among the subjects with pressure injury, 56\% developed initial blanchable redness within $1.84 \pm$ 2.26 mean $\pm \mathrm{SD} d$ and pressure injury within $3.72 \pm 2.72$ mean \pm SD d after placement on NIV. We found that older subjects were more likely to incur pressure injury $(P=.01)$. Underlying chronic disease $(n=232$ [91\%]) was prevalent in this study group. Neither history of chronic illness, sex, the presence of an underlying diagnosis, nor use of NIV-CPAP at home, correlated with pressure injury (Table 1).

Other variables that met statistical significance included maximum inspiratory positive airway pressure $(P=.01)$, the use of more than one mask $(P=.01)$, maximum leak $(P=.01), 95$ th percentile leak $(P=.01)$, and log duration of time on NIV $(P=.01)$. There were no pressure injuries in the 5 CPAP-only episodes. There were 154 of 343 episodes $(44.9 \%)$ in which more than one mask was used. Minimum leak $(P=.054)$, Braden Q score $(P=.08)$, and mean subject mask leak $(P=.95)$ did not reach significance (Table 2). Due to the non-normal distribution of the time on NIV and hospital stay, we also conducted a robust non-parametric analysis of their relationship with ulcer occurrence with clustered rank-sum tests. ${ }^{16}$ Similar to the
Table 3. Multivariable Logistic Mixed Model Output Episode for the Presence of Any Pressure Injury, With Predictors Determined by Conditional AIC

\begin{tabular}{lcccc}
\hline \hline \multicolumn{1}{c}{ Parameter } & Estimate & Standard Error & $P$ & Odds Ratio (95\% CI) \\
\hline Intercept & -4.619 & 1.251 & $<.001$ & NR \\
Maximum leak & 0.021 & 0.009 & .01 & $1.022(1.005-1.039)$ \\
Minimum leak & -0.048 & 0.028 & .09 & $0.953(0.902-1.007)$ \\
Age & 0.08 & 0.047 & .09 & $1.083(0.989-1.187)$ \\
& \\
Logistic mixed model applies to maximum leak, minimum leak, and age with $P<.05$ \\
signifying statistical significance. \\
AIC = Akaike Information Criterion \\
NR = not reported \\
\hline
\end{tabular}

mixed logistic regression, we did not find a significant relationship between either variable, although the time on NIV was borderline $(P=.068)$. After multivariable logistic regression, with age, maximum leak, and minimum leak being identified through the conditional Akaike information criterion, only maximum leak $(P=.01)$ maintained statistical significance (Table 3).

\section{Discussion}

In this retrospective study of subjects treated with NIV at a tertiary-care children's hospital, we determined risk factors that were significantly associated with pressure injury related to NIV use. In our sample, $7.3 \%$ of NIV-use episodes resulted in pressure injury, and $92 \%$ of these were stage 1 or 2 , a less severe designation. In univariate analysis, high mask leak and higher inspiratory posi- 
tive airway pressure pressures were associated with a higher likelihood of developing pressure injury. The use of more than one mask also correlated with pressure injury, which is likely related to good compliance with our policy during the study period to begin alternating masks at the first sign of skin redness. During multivariate analysis, maximum mask leak was the only variable that maintained statistical significance. Determination of risk factors aids identification of patients at higher risk for NIV device-related pressure injuries, which supports the use of protocols that use frequent monitoring of skin integrity, mask rotation, leak assessment, and thoughtful use of high inspiratory positive airway pressure pressures in patients identified as at risk.

Previous studies of adults have similarly identified risk factors for development of pressure injury in hospitalized NIV users. These investigations largely focused on mask-strap tension and positive airway pressure levels. Worsely et al ${ }^{17}$ reported that mask-strap tension impacts patient comfort and nasal contact pressures. Dellweg et al ${ }^{18}$ studied the role of inspiratory and expiratory pressure levels on skin pressure contact and found that increasing inspiratory pressure is associated with higher contact pressure and air leak.

Little has been reported about the relationship between mask leak and pressure injury in pediatric subjects. In our institution, NIV patient-mask leak is measured routinely on hospitalized patients and allowing a small leak is often used to ensure adequate fit and mask position. To the best of our knowledge, this was the first study on NIV users that researched the correlation between leak and pressure injury. Although an inappropriately low leak is hypothesized to be a risk factor for pressure injury due to an overly tight fitting mask, inappropriately high leak conversely was identified as a significant risk factor. High mask patient leak may imply mask dislodgement or an ill-fitting mask, which leads to inappropriately high pressure over a portion of the facial contact area and causes pressure injury.

Although the relationship between the magnitude of NIV pressure and skin-contact pressure has been investigated, ${ }^{15,17}$ less is known regarding the amount of leak that is acceptable and how this relates to the development of pressure injuries. To prevent large leaks, bedside clinicians may tighten interface straps. Although tightening straps can minimize leak, this subsequently increases the contact pressure on the face. ${ }^{17}$ Patient mask leak measurement provides an objective measure that may be useful to guide bedside practitioners to achieve a safe amount of contact pressure while concurrently used as a strategy to decrease the risk of developing pressure injury. There is evidence that ensuring a proper fit to minimize friction and contact pressure ${ }^{2}$ is the best way to prevent pressure injury. Loose straps increase leak, while allowing the interface to shift with patient movement. To prevent pressure injury, the clinician must ensure that the interface mini- mizes contact pressure while maintaining airway pressures. A large leak suggests that a mask is not properly fitting. High mask leak can imply mask dislodgement with skin exposed to subsequent high pressure.

Children who are medically complex have previously been found to be at risk for pressure injury. Cohen et al ${ }^{19}$ identified medically complex children by 4 key features: multiple family-identified service needs, chronic conditions, functional limitations, and high health-care use. The National Institute of Health and Care Excellence has defined young people at risk for pressure injury as those with significant loss of sensation, nutritional deficiency, severe cognitive impairment, or inability to reposition themselves. ${ }^{20}$ Chronic disease was not significantly associated with pressure injury in this study; however, $91 \%$ of the study subjects had an underlying chronic illness.

Limitations to our study deserve comment. Due to the nature of a retrospective study, exposure and outcome assessment could not be controlled, and there was potential for measurement and sampling error. Many of our subjects transitioned from ICUs to wards during the same episode, which made it difficult to extract data based on the area of care. Although many of our respiratory therapists are trained in both intensive and subacute care, not all therapists are, which could lead to detection bias. In our study, we chose to present analysis based on each episode individually. When we performed the same analysis with children individually, we found that the same factors predicted pressure injury. Few subjects in the study had pressure injury, which created a potential for a type- 2 error. As NIV masks change in design, outcomes may vary with newer designs. Data were not collected to assess the use of a barrier with all masks, which could confound the study outcomes.

Having identified maximum leak as statistically significant with the onset of pressure injury in pediatric subjects provided us with a framework for potentially preventing pressure injury based on close observation of mask leak. We speculate that prospective study designs that evaluate the efficacy of maintaining a recommended mask leak interval guideline in children to prevent pressure injuries would be a next step. Larger, prospective investigations can also control for factors known to impact pressure injury. Notwithstanding, multi-disciplinary education regarding mask leak is expected to positively impact management of pediatric NIV.

\section{Conclusions}

The findings from our retrospective study that evaluated the incidence of pressure injury in children prescribed NIV in a hospital-care setting revealed that only high mask leak was significantly associated with pressure injury after con- 


\section{NIV-Related Pressure InJuries in ChildRen}

trol of other pertinent variables. Future prospective study designs should aim to minimize mask leak as a possible therapeutic strategy to eliminate NIV-related pressure injury.

\section{REFERENCES}

1. Cullen Gill E. Reducing hospital acquired pressure ulcers in intensive care. BMJ Qual Improv Rep 2015;4(1). pii: u205599.w3015.

2. Apold J, Rydrych D. Preventing device-related pressure ulcers. J Nurs Care Qual 2012;27(1):28-34

3. Dean JM. Skin health; prevention and treatment of skin breakdown. Transverse Myelitis Assoc J 2011;V:26-31.

4. Shojania KG, Duncan, BW, McDonald, KM, Wachter RM, Markowitz AJ. Making health care safer: a critical analysis of patient safety practices. Evid Rep Technol Assess (Summ) 2001;(43):i-x, 1-668.

5. Jull A. Is pressure sore prevention a sensitive indicator of the quality of nursing care? A cautionary note. Int J Nurs Stud 2010;47(5):531533.

6. The Joint Commission on Accreditation of Healthcare Organization aJCR, Inc. Preventing Pressure Injuries. Joint Commission J Qual Patient Safety 2016(25):1-4.

7. Nightingale F. Notes on nursing: what it is, and what it is not. New York: D. Appleton;1860: 40.

8. Acorda DE. Nursing and respiratory collaboration prevents bipaprelated pressure ulcers. J Pediatr Nurs 2015;30(4):620-623.

9. National Pressure Ulcer Advisory Panel and European Pressure U1cer Advisory Panel. Pressure Ulcer Treatment: Technical Report. Washington DC: Natuional Pressure Ulcer Advisory Panel; 2009. Available at: https://www.npuap.org/wp-content/uploads/2012/03/ Final-2009-Treatment-Technical-Report1.pdf. Accessed on June 12, 2018.

10. The Royal Children's Hospital Melbourne. Pressure Injury Prevention and Management; 2019. Available at: https://www.rch.org.au/ rchcpg/hospital_clinical_guideline_index/pressure_injury_prevention_and_management/. Accessed on June 12, 2018.

11. Haesler E, editor. National Pressure Ulcer Advisory Panel, European Pressure Ulcer Advisory Panel and Pan Pacific Injury Alliance. Prevention and Treatment of Pressure Ulcers: Quick reference guide. Perth, Australia: Cambridge Media; 2014.

12. Noonan C, Quigley S, Curley MA. Using the Braden Q scale to predict pressure ulcer risk in pediatric patients. J Pediatr Nurs 2011; 26(6):566-575.

13. Respironics. Principles of operation. Respironics, Inc, Editor. BiPAP Vision Ventilatory Support System Clinical Manual. Murrysville, PA: Respironics; 2000:3-14.

14. Vaida F, Blanchard, S. Conditional Akaike information for mixedeffects models. Biometrika 2005;92(2):351-370.

15. Saefken B, Rugamer D, Kneib T, and Greven S. Conditional model selection in mixed-effects models with cAIC4. arxiv: 1803.05664v2(stat.co) 2018.

16. Datta S, Satten GA. Rank-Sum Tests for Clustered Data. J Am Stat Assoc 2005;100(471):908-915.

17. Worsely PR, Prudden G, Gower G, Bader DL. Investigating the effects of strap tension during non-invasive ventilation mask application: a combined biomechanical and biomarker approach. Med Devices (Auckl) 2016;9:409-417.

18. Dellweg D, Hochrainer D, Klauke M, Kerl J, Eiger G, Kohler D. Determinants of skin contact pressure formation during non-invasive ventilation. Journal of Biomechanics 2010;43(4):652-657.

19. Cohen E, Kuo DZ, Agrawal R, Berry JG, Bhagat SK, Simon TD, Srivastava R. Children with medical complexity: an emerging population for clinical and research initiatives. Pediatrics 2011;127(3): 529-538.

20. Stansby GAL, Jones K, Marsden G. Prevention and management of pressure ulcers in primary and secondary care: summary of NICU guidance. BMJ 2014;348:g2592.

This article is approved for Continuing Respiratory Care Education credit. For information and to obtain your CRCE

(free to AARC members) visit

www.rcjournal.com

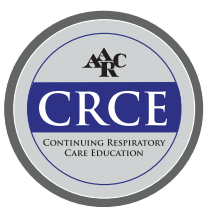

